\title{
Diagnostic accuracy of TB-LAMP for pulmonary tuberculosis: a systematic review and meta-analysis
}

\author{
Priya B. Shete ${ }^{1,2^{*}+} \mathbb{D}$, Katherine Farr ${ }^{1,2 \dagger}$, Luke Strnad ${ }^{3}$, Christen M. Gray ${ }^{4}$ and Adithya Cattamanchi ${ }^{1,2}$
}

\begin{abstract}
Background: The need for a rapid, molecular test to diagnose tuberculosis (TB) has prompted exploration of TB-LAMP (Eiken; Tokyo, Japan) for use in resource-limited settings. We conducted a systematic review to assess the accuracy of TB-LAMP as a diagnostic test for pulmonary TB.

Methods: We analyzed individual-level data for eligible patients from all studies of TB-LAMP conducted between Jan 2012 and October 2015 to compare the diagnostic accuracy of TB-LAMP with that of smear microscopy and Xpert MTB/RIF ${ }^{\oplus}$ using 3 reference standards of varying stringency. Pooled sensitivity and specificity and pooled differences in sensitivity and specificity were estimated using random effects meta-analysis. Study quality was evaluated using QUADAS-2.

Results: Four thousand seven hundred sixty individuals across 13 studies met eligibility criteria. Methodological quality was judged to be low for all studies. TB-LAMP had higher sensitivity than sputum smear microscopy (pooled sensitivity difference $+13 \cdot 2,95 \% \mathrm{Cl} 4 \cdot 5-21.9 \%$ ) and similar sensitivity to Xpert MTB/RIF (pooled sensitivity difference $-2 \cdot 5,95 \% \mathrm{Cl}$ -8.0 to +2.9$)$ using the most stringent reference standard available. Specificity of TB-LAMP was similar to that of sputum smear microscopy (pooled specificity difference $-1.8,95 \% \mathrm{Cl}-3.8$ to +0.2 ) and Xpert MTB/RIF (pooled specificity difference $0.5,95 \% \mathrm{Cl}-0.9$ to $+1 \cdot 8)$.

Conclusions: From the perspective of diagnostic accuracy, TB-LAMP may be considered as an alternative test for sputum smear microscopy. Additional factors such as cost, feasibility, and acceptability in settings that continue to rely on sputum smear microscopy should be considered when deciding to adopt this technology. Xpert MTB/ RIF should continue to be preferred in settings where resource and infrastructure requirements are adequate and where HIV co-infection or drug-resistance is of concern.
\end{abstract}

Keywords: Tuberculosis, Diagnostic testing, Molecular assay, Point of care

\section{Background}

Better diagnostics are essential for achieving global tuberculosis (TB) elimination targets. In 2013, Xpert MTB/RIF ${ }^{\bullet}$ (Xpert) (Cepheid, Sunnyvale, CA, USA) became the first molecular TB test endorsed by the World Health Organization (WHO), and there has

\footnotetext{
* Correspondence: Priya.shete@ucsf.edu

${ }^{\dagger}$ Priya B. Shete and Katherine Farr contributed equally to this work.

${ }^{1}$ Division of Pulmonary and Critical Care Medicine, University of California

San Francisco and Zuckerberg San Francisco General Hospital, 1001 Potrero

Avenue, 5K1, San Francisco, CA 94110, USA

${ }^{2}$ Curry International Tuberculosis Center, University of California San

Francisco, San Francisco, CA, USA

Full list of author information is available at the end of the article
}

since been considerable investment in its scale-up [1]. However, relatively high device and consumable costs, infrastructure requirements, and need for continuous instrument maintenance remain obstacles to use of Xpert as a point-of-care test in peripheral health centers where the majority of $\mathrm{TB}$ patients initially present for care.

To expand the availability of molecular testing for TB, Eiken Chemical Co., Ltd. developed a commercial version of loop-mediated isothermal amplification (LAMP), a technique in which nucleic acid amplification occurs under isothermal conditions. LAMP is based on auto-cycling, strand displacement DNA synthesis 
performed by a DNA polymerase with high strand displacement activity and two specially designed inner and two outer primers [2,3]. Eiken's Loopamp MTBC Detection Kit (TB-LAMP) targets the gyrB and IS regions of the Mycobacterium tuberculosis (MTB) complex genome. Detection of amplified product is based on turbidity visualized with the naked eye or under ultraviolet (UV) light after 15$60 \mathrm{~min}$ [4-7].

To inform WHO guideline development, we conducted a systematic review of studies evaluating the diagnostic accuracy of TB-LAMP using the latest assay kit and protocol. The primary objective was to evaluate the diagnostic accuracy of TB-LAMP if used as an alternative test for sputum smear microscopy among adults suspected of having pulmonary TB. In addition, we sought to determine the diagnostic accuracy of TB-LAMP if used as an alternative test for microscopy among adults with HIV infection or as an add-on test for adults with negative sputum smear microscopy results, and the proportion of indeterminate/invalid TB-LAMP results.

\section{Methods}

We followed standard guidelines and methods for systematic reviews and meta-analyses of diagnostic tests [8]. We developed four PICO style research questions to inform this review. First, what is the diagnostic accuracy of TB-LAMP for detection of pulmonary $\mathrm{TB}$ in reference to mycobacterial culture if used as an alternative test for sputum smear microscopy among all adults and among HIV-infected adults? Second, what is the diagnostic accuracy of TB-LAMP for detection of pulmonary TB in reference to mycobacterial culture if used as an add-on test among sputum smear-negative adults? Third, what is the difference in diagnostic accuracy between TB-LAMP and Xpert for detection of pulmonary $\mathrm{TB}$ in reference to mycobacterial culture among all adults? And finally, what is the proportion of indeterminate/invalid results when TB-LAMP is used to detect pulmonary $\mathrm{TB}$ among all adults and among HIV-infected adults?

\section{Search strategy}

To perform a study of TB-LAMP, investigators must order kits directly from Eiken. Therefore, a list of such studies was requested from Eiken. To confirm the list provided was complete, we performed a search in Google Scholar and PubMed using the terms "TB LAMP", "TB-LAMP", and "tuberculosis LAMP". To meet the deadline for WHO guideline development in January 2016, we only included studies completed by October 1, 2015.

\section{Study and participant selection}

We included all studies which 1) evaluated the Eiken TB-LAMP kit on sputum samples from adult presumptive TB patients; 2) were conducted in an intermediate or high TB burden country as defined by WHO; and 3) were conducted after January 1, 2012 using the final protocol, training, and TB-LAMP kits approved by Eiken. We excluded studies that 1) did not exclude patients on TB treatment within 60 days of enrollment; 2) did not perform speciation testing to confirm presence of Mycobacterium tuberculosis (MTB) complex in positive cultures; or 3) performed TB-LAMP on frozen specimens.

Authors of eligible studies provided individual participant data. We excluded individual participants who were 1) less than 18 years of age; 2) did not have results of speciation testing for MTB; 3 ) had a positive culture but speciation testing identified only non-tuberculous mycobacteria (NTM); 4) had a documented history of prior TB; 5) had TB-LAMP testing performed on non-sputum samples; 6) had TB-LAMP testing done with a total reaction volume of $<25 \mu \mathrm{L}$; or 7 ) could not be classified as TB-positive or TB-negative based on the reference standard definitions described below. When comparing TB-LAMP to Xpert, we also excluded individual participants for whom Xpert was performed on frozen samples or valid results were unavailable for both TB-LAMP and Xpert.

\section{Quality assessment}

We used the Assessment of Diagnostic Accuracy Studies (QUADAS-2) tool to assess the methodological quality of eligible studies [9]. Specific yes/no signaling questions were tailored for each QUADAS-2 domain.

\section{Index tests}

Studies recorded LAMP and Xpert results as negative, positive or indeterminate/invalid in accordance with manufacturer recommendations. We standardized sputum smear microscopy results across studies by considering only direct ZN (Ziehl-Neelsen) and direct FM (fluorescence microscopy) results, considering only the first two smear results if more than two direct $\mathrm{ZN}$ or direct FM results were available, and defining patients to be sputum smear-positive if $\geq 1$ acid-fast bacillus (AFB) was identified in any sputum smear.

\section{Reference standard}

We used three hierarchical mycobacterial culture-based reference standards to account for differences in the number of cultures performed and results available. Patients were classified as having TB if one or more cultures were positive and confirmed to be MTB complex by speciation testing. Patients were classified as not 
having TB if there were no positive cultures and at least 1) two negative cultures performed on different sputum samples (Standard 1); 2) two negative cultures performed on one sputum sample (Standard 2); or 3) one negative culture (Standard 3). The three reference standards allowed for a trade-off between yield of TB diagnosis (highest with Standard 1 and lowest with Standard 3) and number of studies/participants included in each analysis (lowest with Standard 1 and highest with Standard 3).

\section{Statistical analysis}

For all review questions, we assessed heterogeneity visually with forest plots and statistically with the $x^{2}$ test for heterogeneity and the $I^{2}$ test of inconsistency $[10,11]$. When four or more studies were available, we generated pooled summary estimates of sensitivity/ specificity using hierarchical summary receiver operating characteristic (HSROC) analysis. We plotted these estimates along with 95\% confidence regions in ROC space. Pooled differences in sensitivity/specificity between TB-LAMP and Xpert and pooled proportion of indeterminate/invalid TB-LAMP results were generated using random effects meta-analysis. For the primary review question of TB-LAMP accuracy if used as an alernative test for smear microscopy, we explored potential reasons for heterogeneity by performing sub-group analyses based on the health system level (reference lab, microscopy center, or hospital-affiliated clinics) at which the study was conducted and study quality (high-quality studies across all domains and within each domain of QUADAS-2).

\section{Results}

Of 20 studies identified by Eiken at the time of this review, 13 met criteria for inclusion (Fig. 1): four evaluation (EVAL) studies were conducted by the Foundation for Innovative New Diagnostics (FIND) in reference labs; one demonstration (DEMO) study was

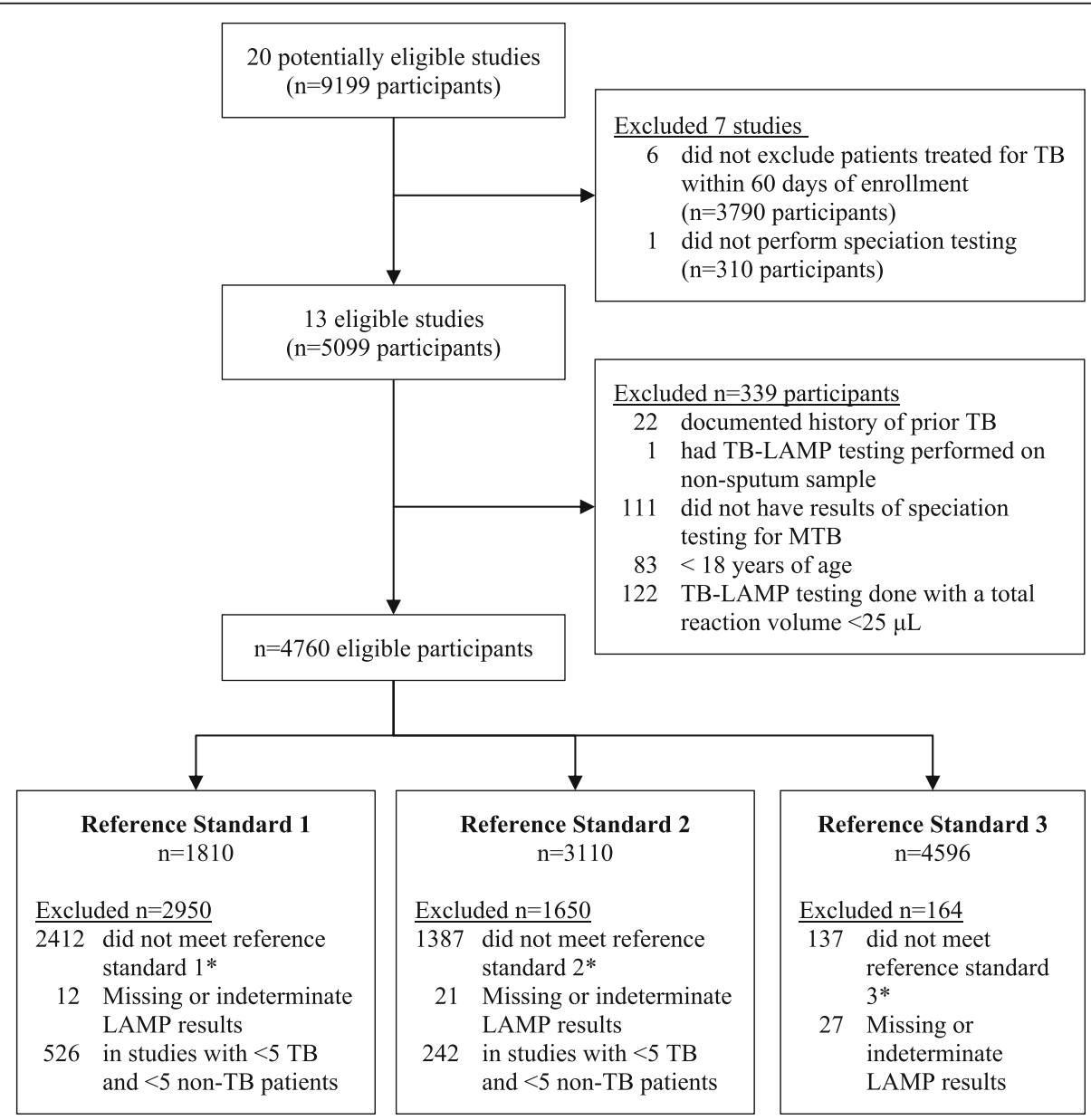

Fig. 1 Study and participant selection flow diagram. Of 20 potentially eligible studies, 13 met study-level eligibility criteria. The 13 eligible studies included 5099 participants, of whom 339 (7\%) did not meet participant-level eligibility criteria. Of the 4760 eligible participants, 1810 (38\%) were included in the analysis for reference standard 1, 3110 (65\%) for reference standard 2, and 4596 (97\%) for reference standard 3. Abbreviations: TB, Tuberculosis; MTB, Mycobacterium tuberculosis; $\mu \mathrm{L}$, microliter 
conducted by FIND in a peripheral microscopy center; seven studies were commissioned by FIND through a request for applications (RFA); and one study was sponsored by Eiken [12-14]. Authors of included studies submitted individual data for 5099 participants, of whom 4760 were eligible for analysis (Additional file 1: Table S1).

Of the included studies, four were conducted at reference laboratories, six at hospital and/or university-affiliated clinics, and three at peripheral microscopy centers (Table 1). Study participants were majority male with a median age of 40 (IQR 29-54) years. More than $10 \%$ of participants were known to be HIV-positive in four of 13 studies (S. Africa EVAL, Malawi RFA, Uganda RFA, Ivory Coast RFA). The proportion of patients with culture-positive TB was 20$40 \%$ in most studies, but was notably lower (8-15\%) in three studies (India DEMO, India RFA, Vietnam RFA) and higher (66\%) in one study (Vietnam EVAL). The proportion of patients with smear-negative TB ranged widely from 13 to $59 \%$.

\section{Methodological quality}

We considered overall risk of bias to be high due to problems with the culture-based reference standard (all 13 studies), unclear patient selection (five studies), and flow and timing concerns (eight studies) (Fig. 2). We found applicability concerns to be low for index tests and the reference standard; however, we judged five studies to have high applicability concerns for patient selection because they were conducted at referral laboratories/centers or because enrollment involved screening of patients by a pulmonary specialist (Additional file 1 : Table S2).

\section{TB-LAMP accuracy if used as an alternative test for smear microscopy}

Sensitivity of TB-LAMP in individual studies ranged from 66 to 91\% with Standard 1 (Fig. 3), 62-91\% with Standard 2 and $48-100 \%$ with Standard 3 (Additional file 1: Figure S1). We found significant heterogeneity in sensitivity estimates, both from visual inspection of forest plots and statistical testing $\left(I^{2}\right.$ $72-94 \%, p<0.003$ for all reference standards). Pooled sensitivity of TB-LAMP ranged from $77.7 \%$ (95\% CI 71.2-83.0) when using Standard 1 to $80.3 \%$ (95\% CI 70.3-87.5) when using Standard 3 (Table 2 and Additional file 1: Figure S2). TB-LAMP was more sensitive than sputum smear microcopy, with the sensitivity difference ranging from $7.1 \%$ (95\% CI 1.412.9) when using Standard 1 to $13.2 \%$ (95\% CI $4.5-$ 21.9) when using Standard 3.

Specificity of TB-LAMP in individual studies ranged from 90 to $99 \%$ with Standard 1 (Fig. 3), and 90-100\% with Standards 2 and 3 (Additional file 1: Figure S1). Visual inspection of forest plots indicated heterogeneity in specificity estimates was less than for sensitivity estimates, but was still significant $\left(I^{2} 61-78 \%, p<0.03\right.$ for all reference standards). Pooled specificity of TB-LAMP ranged from 97.7\% (95\% CI 96.1-98.7) when using Standard 3 to $98.1 \%$ (95\% CI 95.7-99.2) when using Standard 1 (Table 2 and Additional file 1: Figure S2). TB-LAMP had similar specificity to sputum smear microscopy, with specificity difference ranging from $-1.8 \%$ (95\% CI -3.8-0.2) when using Standard 1 to $-1.3 \%$ (95\% CI $-3 \cdot 1 \%$ to +0.4 ) when using Standard 3.

Sub-group analyses did not reduce heterogeneity in pooled sensitivity and specificity estimates. Pooled sensitivity was $78.0 \%$ (95\% CI 68.9-85.0) among studies conducted in reference labs and $86.8 \%$ (95\% CI 67.9-95.4) among studies conducted in hospital-affiliated clinics, and there was significant heterogeneity within both sub-groups $\left(I^{2} 74-96 \%, p<0.01\right.$ for all reference standards) (Additional file 1: Table S3). Among studies rated as high-quality for patient selection, pooled sensitivity was $84.2 \%$ (95\% CI $71.1-92.0)$ and pooled specificity 98.1\% (95\% CI 94.3-99.4), but there was significant heterogeneity across studies $\left(I^{2} 83 \%, \mathrm{p}<0.001\right.$ for sensitivity and $I^{2} 87 \%, \mathrm{p}<0.001$ for specificity). Pooled estimates could not be obtained for studies conducted at microscopy centers and studies rated as high-quality for reference standard because there were less than four studies in these sub-groups.

\section{TB-LAMP accuracy if used as an alternative test for smear microscopy in HIV-infected adults}

Pooled sensitivity of TB-LAMP was lower among HIV-infected adults than all adults, ranging from 63.8\% (95\% CI 49.0-76.4) with Standard 2 to 73.4 (95\% CI 51.9-87.6) with Standard 3 (Table 2 and Additional file 1: Figure S3). Pooled specificity was low with Standard 3 (95.0, 95\% CI 64.0-99.5) but high with Standard 2 (98.8, 95\% CI 85.1-99.9). There was considerable heterogeneity in sensitivity $\left(I^{2} 86 \%\right.$, $p<0.001)$ and specificity $\left(I^{2} 86 \%, \mathrm{p}<0.001\right)$ estimates with Standard 3, but not Standard $2\left(I^{2} 54 \%, p=0.09\right.$ for sensitivity and $I^{2} 0 \%, \mathrm{p}=0.42$ for specificity) (Fig. 3). There were insufficient studies $(N=2)$ to perform meta-analysis using Standard 1.

\section{TB-LAMP accuracy if used as an add-on test in smear- negative adults}

As expected, pooled sensitivity of TB-LAMP was lower among smear-negative adults than among all adults, ranging from $40.3 \%$ (95\% CI 27.9-54.0) with Standard 3 to $42.2 \%$ (95\% CI 27.9-57.9) with Standard 2 (Table 2 and Additional file 1: Figure S4). Pooled specificity of TB-LAMP among smear-negative adults was similar to 


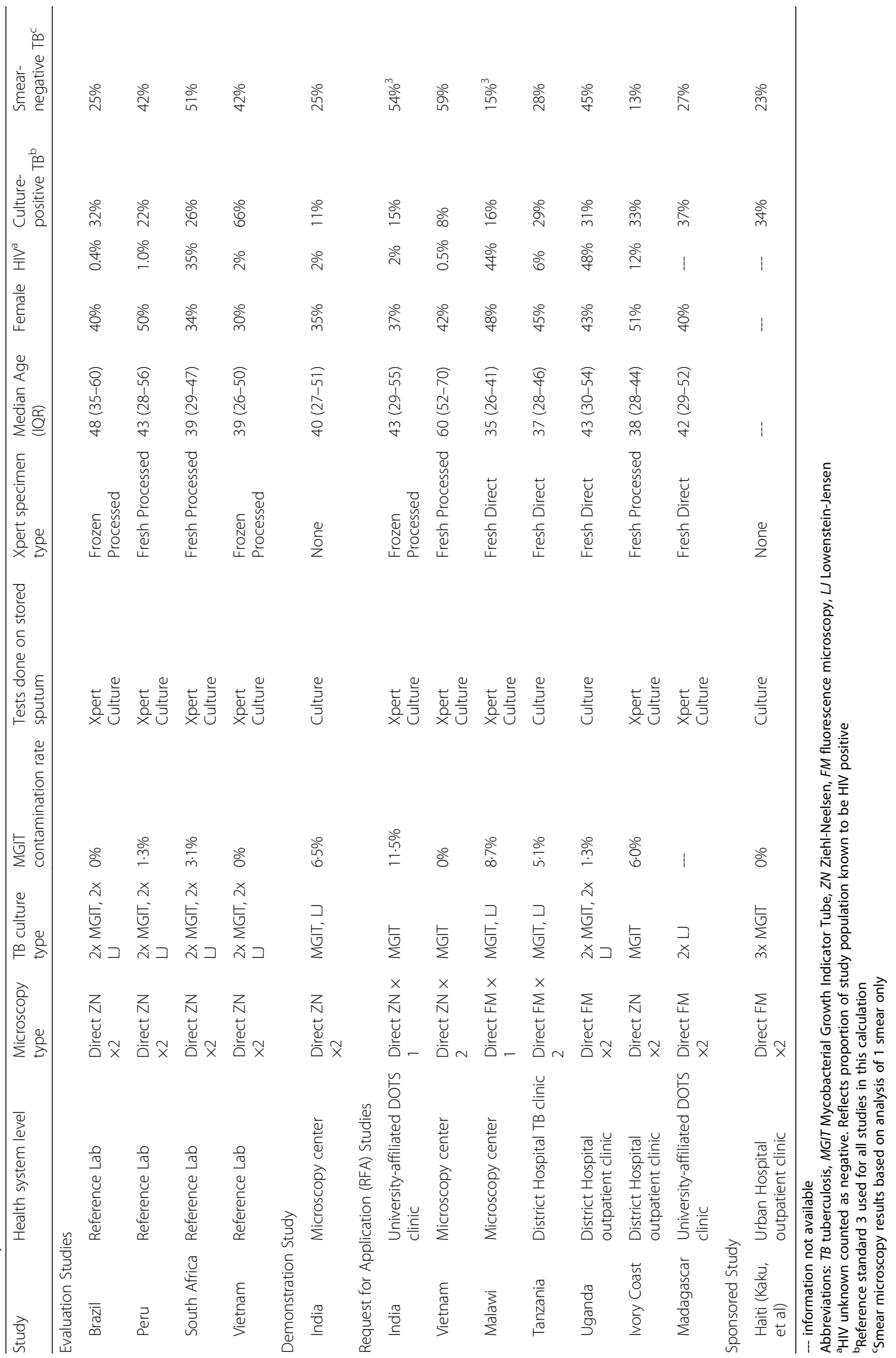




\begin{tabular}{|c|c|c|c|c|c|c|c|}
\hline & \multicolumn{4}{|c|}{ Risk of Bias } & \multicolumn{3}{|c|}{$\begin{array}{l}\text { Applicability } \\
\text { Concerns }\end{array}$} \\
\hline & 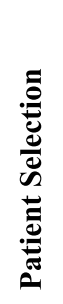 & $\bar{\Xi}$ & 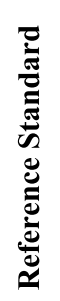 & 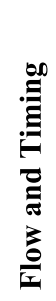 & 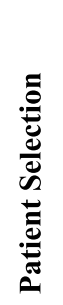 & 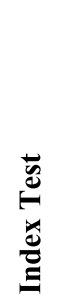 & 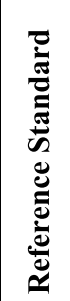 \\
\hline Brazil (EVAL) & + & & & + & & & \\
\hline Peru (EVAL) & 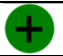 & & & + & & & 1. \\
\hline South Africa (EVAL) & + & & & + & & & + \\
\hline Vietnam (EVAL) & 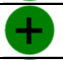 & & & + & - & & \\
\hline India (DEMO) & + & & & + & 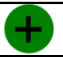 & & 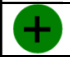 \\
\hline India (RFA) & $?$ & & & $?$ & + & & + \\
\hline Vietnam (RFA) & $?$ & & & $?$ & + & & + \\
\hline Malawi (RFA) & + & & & $(-)$ & & & \\
\hline Tanzania (RFA) & $?$ & 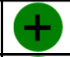 & 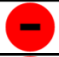 & $?$ & - & & 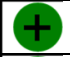 \\
\hline Uganda (RFA) & $?$ & & $=$ & $?$ & 4 & & \\
\hline Ivory Coast (RFA) & + & & - & $?$ & + & & \\
\hline Madagascar (RFA) & + & 1 & $=$ & $?$ & + & & \\
\hline Haiti (Kaku et al) & $?$ & 16 & 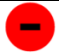 & $?$ & + & & 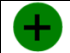 \\
\hline
\end{tabular}

Low risk of bias

? Unclear risk of bias

High risk of bias

Fig. 2 Study quality assessment using QUADAS-2. Based on QUADAS-2 assessment, the risk of bias was judged to be unclear in 5 (38\%) studies due to patient selection issues, high for all studies due to an inadequate reference standard, and either high in 1 (8\%) study or unclear in 7 studies (54\%) due to flow and timing issues. In addition, applicability concerns were high in 5 (38\%) studies due to patient selection issues

that observed among all adults, ranging from 97.7\% (95\% CI 96.1-98.6) with Standard 3 to 98.4\% (95\% CI 95.9-99.4) with Standard 1. There was greater heterogeneity in sensitivity estimates than in specificity estimates across studies (Fig. 3).

\section{Comparison of diagnostic accuracy of TB-LAMP and Xpert}

Xpert sensitivity across individual studies ranged from 65 to $97 \%$ between the three reference standards and specificity ranged from 90 to $100 \%$ (Additional file 1: Figure S5). TB-LAMP sensitivity ranged from 48 to 93\% between reference standards, and specificity ranged from 94 to $100 \%$ (Additional file 1: Figure S6). The difference in sensitivity between TB-LAMP and Xpert in individual studies ranged from -14 to $+3 \%$ for Standard 1, -15 to $+3 \%$ for Standard 2, and -36 to $+3 \%$ for Standard 3 (Additional file 1: Figure S7). TB-LAMP had similar sensitivity compared to Xpert using Standard 1 (pooled sensitivity difference $-2.5 \%$
(95\% CI -8.0 to +2.9$)$ but lower sensitivity when using Standard $3(-6.9,95 \%$ CI -12.8 to -1.0$)$ (Table 3). Difference in specificity between TB-LAMP and Xpert in individual studies ranged from -1 to $+3 \%$ for Standard 1, -1 to $+4 \%$ for Standard 2, and -3 to $+5 \%$ for Standard 3 (Additional file 1: Figure S8). There was no difference in specificity of TB-LAMP and Xpert regardless of reference standard used (Table 3). Heterogeneity varied depending on the reference standard used, with minimal heterogeneity in sensitivity and specificity differences across studies with Standard 1, and significant heterogeneity with Standard 3 (Additional file 1: Figure S7 and Figure S8).

\section{Indeterminate/invalid TB-LAMP results}

The proportion of indeterminate TB-LAMP results was $0 \%$ in 11 studies and $1 \%$ in two studies (Additional file 1 : Figure S9). There was minimal heterogeneity across studies $\left(I^{2} \quad 28 \%, \quad p=0 \cdot 25\right)$. Pooled proportion of 


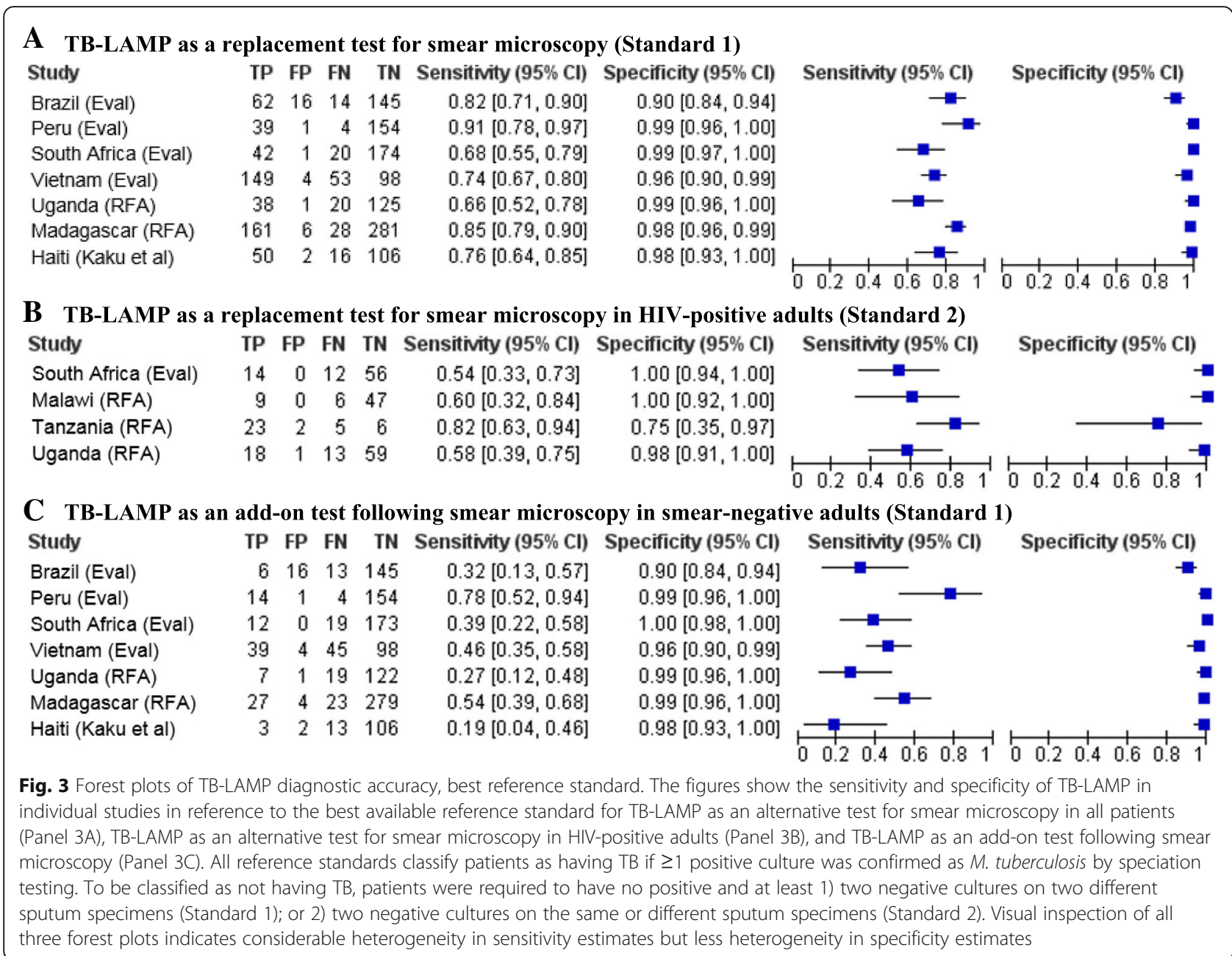

indeterminate TB-LAMP results was $<0.1 \%$ (95\% CI 0-0). Results were similar among HIV-infected adults; pooled proportion of indeterminate TB-LAMP results in this sub-group was $<0.1 \%$ (95\% CI 0-1).

\section{Discussion}

This systematic review identified 13 studies conducted in intermediate to high TB burden countries evaluating the accuracy of TB-LAMP performed directly on sputum samples for diagnosis of pulmonary TB. TB-LAMP had moderate sensitivity (pooled sensitivity 77.7, 95\% CI 71.2-83.0) and high specificity (98.1, 95\% CI 95.7-99.2) when using the most stringent culture-based reference standard. Sensitivity was lower among HIV-infected adults (pooled sensitivity 63.8, 95\% CI 49.0-76.4), likely due to a higher proportion of smear-negative TB in this population. Among all adults, TB-LAMP would identify slightly less than half of all smear-negative TB patients (pooled sensitivity $42.1,95 \%$ CI $30 \cdot 0-55 \cdot 3$ ) if used as an add-on test following sputum smear microscopy. Diagnostic accuracy was comparable to that of Xpert, with no significant differences in pooled sensitivity $(-2 \cdot 5$, $95 \% \mathrm{CI}-8.0$ to +2.9$)$ or pooled specificity $(0.5,95 \% \mathrm{CI}$ -0.9 to +1.8$)$ using the most stringent reference standard. Finally, this review found indeterminate TB-LAMP results were extremely uncommon (pooled proportion < 0.1, 95\% CI 0-0) (Additional file 1: Figure S9). Overall, these data support a potential role for TB-LAMP in the diagnosis of pulmonary $\mathrm{TB}$ in intermediate- to high-burden countries where smear-microscopy is still the predominant mode of TB diagnosis.

The target product profile for an alternative test for smear microscopy recommends a sensitivity of at least $80 \%$ and specificity of at least $98 \%$ [15]. TB-LAMP very nearly meets these criteria, although sensitivity was below the recommended minimum of $60 \%$ for smear-negative TB and $99 \%$ for smear-positive TB. Nonetheless, TB-LAMP was consistently more sensitive than sputum smear microscopy in individual studies, and pooled sensitivity difference was $7 \cdot 1 \%-13 \cdot 2 \%$ in favor of TB-LAMP depending on reference standard used. Thus, it can be expected that use of TB-LAMP as 
Table 2 Pooled sensitivity and specificity of TB-LAMP

\begin{tabular}{|c|c|c|}
\hline Reference Standard & Pooled Sensitivity & Pooled Specificity \\
\hline \multicolumn{3}{|c|}{$\begin{array}{l}\text { TB-LAMP accuracy if used as an alternative test for smear microscopy in } \\
\text { all adults }\end{array}$} \\
\hline Standard $1^{\mathrm{a}}$ & $77.7(71 \cdot 2-83 \cdot 0)$ & $98.1(95.7-99.2)$ \\
\hline Standard $2^{\mathrm{a}}$ & $76.0(69.9-81 \cdot 2)$ & $98.0(96.0-99.0)$ \\
\hline Standard $3^{\mathrm{a}}$ & $80 \cdot 3(70 \cdot 3-87.5)$ & $97.7(96 \cdot 1-98.7)$ \\
\hline \multicolumn{3}{|c|}{$\begin{array}{l}\text { TB-LAMP accuracy if used as an alternative test for smear microscopy in } \\
\text { HIV-positive adults }\end{array}$} \\
\hline Standard $1^{\mathrm{a}}$ & N/A (<4 studies) & N/A (<4 studies) \\
\hline Standard $2^{\mathrm{a}}$ & $63.8(49 \cdot 0-76.4)$ & $98.8(85.1-99.9)$ \\
\hline Standard $3^{\mathrm{a}}$ & $73.4(51.9-87.6)$ & $95.0(64.0-99.5)$ \\
\hline \multicolumn{3}{|c|}{ TB-LAMP accuracy if used as an add-on test in smear-negative adults } \\
\hline Standard $1^{\text {a }}$ & $42 \cdot 1(30 \cdot 0-55 \cdot 3)$ & $98.4(95.9-99.4)$ \\
\hline Standard $2^{\mathrm{a}}$ & $42.2(27.9-57.9)$ & $98.0(96.0-99.0)$ \\
\hline Standard $3^{\mathrm{a}}$ & $40 \cdot 3(27.9-54 \cdot 0)$ & $97 \cdot 7(96 \cdot 1-98 \cdot 6)$ \\
\hline \multicolumn{3}{|c|}{ TB-LAMP accuracy in studies comparing to Xpert $^{\text {b }}$} \\
\hline Standard $1^{\mathrm{a}}$ & $78.0(66 \cdot 6-86 \cdot 4)$ & $98.9(97.4-99.6)$ \\
\hline Standard $2^{\mathrm{a}}$ & $74 \cdot 1(64 \cdot 1-82 \cdot 2)$ & $98.8(96.8-99.6)$ \\
\hline Standard $3^{\mathrm{a}}$ & $75 \cdot 8(63.2-85 \cdot 0)$ & $98.2(96.0-99 \cdot 2)$ \\
\hline \multicolumn{3}{|c|}{ Xpert accuracy in studies comparing to TB-LAMPb } \\
\hline Standard $1^{\mathrm{a}}$ & $81 \cdot 1(70 \cdot 6-88 \cdot 5)$ & $98.2(95.9-99.2)$ \\
\hline Standard $2^{a}$ & $80.4(73.4-85.9)$ & $97.4(94.9-98.7)$ \\
\hline Standard $3^{\mathrm{a}}$ & $84.0(75 \cdot 6-90 \cdot 0)$ & $97.2(94.4-98.6)$ \\
\hline
\end{tabular}

${ }^{a}$ All reference standards classify patients as having TB if $\geq 1$ positive culture was confirmed as $M$. tuberculosis by speciation testing. To be classified as not having $T B$, patients were required to have no positive and at least 1 ) two negative cultures on two different sputum specimens (Standard 1); 2) two negative cultures on the same or different sputum specimens (Standard 2); or 3) at least one negative culture (Standard 3)

${ }^{\mathrm{b}}$ Data restricted to study participants who had valid results for both TB-LAMP and Xpert and testing performed on non-frozen specimens Abbreviations: N/A not applicable

an alternative for sputum smear microscopy would lead to more $\mathrm{TB}$ cases being identified while keeping false-positive results to an acceptable minimum. As discussed further below, a better reference standard would have likely further increased difference in sensitivity and

Table 3 TB-LAMP versus Xpert: Pooled Sensitivity and Specificity differences

\begin{tabular}{lll}
\hline $\begin{array}{l}\text { Reference } \\
\text { standard }\end{array}$ & $\begin{array}{l}\text { Pooled sensitivity } \\
\text { difference }\end{array}$ & $\begin{array}{l}\text { Pooled specificity } \\
\text { difference }^{\mathrm{b}}\end{array}$ \\
\hline Standard $1^{\text {a }}$ & $-2.5(-8.0$ to +2.9$)$ & $0.5(-0.9$ to +1.8$)$ \\
${\text { Standard } 2^{\text {a }}}_{\text {Standard } 3^{\text {a }}}$ & $-6.0(-12.1$ to +0.1$)$ & $1.0(-0.3$ to +2.4$)$ \\
& $-6.9(-12.8$ to -1.0$)$ & $1.1(-0.7$ to +2.8$)$
\end{tabular}

${ }^{a}$ All reference standards classify patients as having TB if $\geq 1$ positive culture was confirmed as $M$. tuberculosis by speciation testing. To be classified as not having $T B$, patients were required to have no positive and at least 1) two negative cultures on two different sputum specimens (Standard 1); 2) two negative cultures on the same or different sputum specimens (Standard 2); or 3) at least one negative culture (Standard 3)

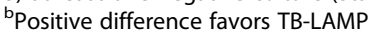

minimized any difference in specificity between TB-LAMP and smear microscopy.

Although more accurate than microscopy, pooled sensitivity of TB-LAMP is lower than has been reported for Xpert (89, 95\% CI 85-92] [16]. However, in head-to-head comparisons, the sensitivity difference between TB-LAMP and Xpert was not statistically significant except when using the least stringent reference standard. Although more data is needed to confirm whether TB-LAMP is as sensitive as Xpert, it is important to consider TB-LAMP has a different end-user profile. It is less costly to deploy and has fewer infrastructure requirements (e.g., stable power), but has higher training requirements due to less automation. Even if confirmed to be less sensitive than Xpert, TB-LAMP may have a role at health centers that have personnel with adequate technical skills but insufficient resources or infrastructure to deploy Xpert. In addition, the specificity of TB-LAMP was as high or higher than that of Xpert, further supporting its use as an alternative test for microscopy in settings without access to Xpert.

There are several limitations to the evidence identified in this review. Most significantly, an inadequate reference standard was used across all studies, likely leading to classification of patients with $\mathrm{TB}$ as not having $\mathrm{TB}$. With a better reference standard, it can be expected that some false-positive TB-LAMP results would be re-classified as true positives, leading to improved sensitivity and specificity. Some true negative TB-LAMP results could also be re-classified as false negatives, leading to lower sensitivity and specificity. Our findings suggest the former is more likely, given that TB-LAMP specificity improved with a more stringent reference standard (i.e., when moving from Standard 3 to Standard 1). A better reference standard may also have increased the sensitivity difference while further minimizing the specificity difference observed between Xpert and TB-LAMP due to the higher number of false-positive Xpert results. Another key limitation is that the included studies may not accurately reflect the introduction of TB-LAMP under programmatic conditions. Only 3 studies were conducted at peripheral health centers and Eiken provided extensive training to sites included in all studies. Further operational research is needed to characterize TB-LAMP performance under typical implementation conditions as an alternative test for microscopy at peripheral health centers. Finally, the data used in this analysis was taken from studies sponsored by either a non-governmental organizations (NGOs) with interest in the product or the manufacturer (Eiken), raising potential concerns about bias due to conflict of interest. To overcome this limitation, this systematic review and meta-analysis were the result of independent analysis of individual level data taken from these studies. PICO 
questions and reference standards were devised by the authors in conjunction with the World Health Organization as part of their guideline development process.

In summary, this systematic review supports use of TB-LAMP as a potential alternative test for smear microscopy for diagnosis of pulmonary TB in intermediateto high-burden countries, particularly in settings where Xpert testing is not feasible. The results of this review have influenced development of WHO guidelines related to the use of TB-LAMP, which now conditionally recommend the use of TB-LAMP as an alternative test for smear microscopy or as an add-on test for smear negative patients, but do not recommend its use in settings where molecular testing such as Xpert is readily available [17]. However, additional studies following standardized protocols and including a high-quality reference standard (liquid culture results on at least two samples) are needed to better inform National TB Programmes of the relative performance of TB-LAMP versus Xpert in programmatic settings. Cost effectiveness analysis conducted as part of WHO guideline development on the use of TB-LAMP demonstrated lower per test cost, operational costs, budgetary costs and favorable incremental cost effectiveness ratios compared to Xpert in a few countries [18]. As with feasibility assessments, additional cost and cost effectiveness analyses in programmatic settings are required to further inform context-specific uptake of TB-LAMP. The evidence to date, along with increased automation and the ability to identify rifampin resistance, suggests Xpert should remain the preferred diagnostic when sufficient financial resources and infrastructure can support its use.

\section{Conclusions}

Although the performance of TB-LAMP has been evaluated in several studies worldwide with variable results, we report the first standardized evaluation of the results of these individual studies to inform policy guidance. The findings of our systematic review and meta-analysis show that TB-LAMP has the potential to be a useful diagnostic test for pulmonary $\mathrm{TB}$, but that additional high quality studies in programmatic conditions should be done to strengthen the case for its use. TB-LAMP performed better than sputum smear microscopy (more sensitive and as specific) in the diagnosis of pulmonary tuberculosis and performed similar to Xpert (similar sensitivity and specificity). The results of this study provided the basis for the WHO's guidelines on the use of TB-LAMP for the diagnosis of pulmonary TB, which recommend that TB-LAMP can be used as an alternative for microscopy for the diagnosis of pulmonary $\mathrm{TB}$ in adults, and can be considered as an add-on test to microscopy particularly in sputum smear-negative adults with TB symptoms in settings where Xpert testing is not available and where drug-resistance or HIV co-infection are not of concern [17].

\section{Additional file}

Additional file 1: Figure S1. Forrest plots of TB-LAMP diagnostic accuracy, additional reference standards. The figures show the sensitivity and specificity of TB-LAMP in individual studies in reference to all additional reference standards not judged best available for TB-LAMP as an alternative test for smear microscopy in all patients (Panel S1A and Panel S1B), TB-LAMP as an alternative test for smear microscopy in HIV-positive adults (Panel S1C), and TB-LAMP as an add-on test following smear microscopy (Panel S1D and Panel $\mathrm{S} 1 \mathrm{E})$. All reference standards classify patients as having TB if $\geq 1$ positive culture was confirmed as M. tuberculosis by speciation testing. To be classified as not having $T B$, patients were required to have no positive and at least 1) two negative cultures on two different sputum specimens (Standard 1); 2) two negative cultures on the same or different sputum specimens (Standard 2); or 3) at least one negative culture (Standard 3). Visual inspection of all three forest plots indicates considerable heterogeneity in sensitivity estimates but less heterogeneity in specificity estimates. Figure S2. TB-LAMP as an alternative for sputum smear microscopy: Summary Receiver Operating Characteristic (SROC) curves. The figure shows SROC curves for TB-LAMP (green line), individual study estimates (grey circle), pooled estimates (red square), and the 95\% confidence region for pooled estimates (yellow dotted line) when using 3 culture-based reference standards. All reference standards classify patients as having TB if $\geq 1$ positive culture was confirmed as $M$. tuberculosis by speciation testing. To be classified as not having TB, patients were required to have no positive and at least 1) two negative cultures on two different sputum specimens (Standard 1); 2) two negative cultures on the same or different sputum specimens (Standard 2); or 3) at least one negative culture (Standard 3). Figure S3. TB-LAMP as an alternative test for smear microscopy in HIV-positives: Summary Receiver Operating Characteristic (SROC) curves. The figure shows SROC curves for TBLAMP (green line), individual study estimates (grey circle), pooled estimates (red square), and the $95 \%$ confidence region for pooled estimates (yellow dotted line) when using 2 culture-based reference standards (no studies qualified for Standard 1). All reference standards classify patients as having TB if $\geq 1$ positive culture was confirmed as M. tuberculosis by speciation testing. To be classified as not having TB, patients were required to have no positive and at least 1) two negative cultures on the same or different sputum specimens (Standard 2); or 2) at least one negative culture (Standard 3). Figure S4. TB-LAMP as an add-on test following smear microscopy: Summary Receiver Operating Characteristic (SROC) curves. The figure shows SROC curves for TBLAMP (green line), individual study estimates (grey circle), pooled estimates (red square), and the $95 \%$ confidence region for pooled estimates (yellow dotted line) when using 3 culture-based reference standards. All reference standards classify patients as having TB if $\geq 1$ positive culture was confirmed as $M$. tuberculosis by speciation testing. To be classified as not having TB, patients were required to have no positive and at least 1) two negative cultures on two different sputum specimens (Standard 1); 2) two negative cultures on the same or different sputum specimens (Standard 2); or 3) at least one negative culture (Standard 3). Figure S5. TB-LAMP vs. Xpert MTB/RIF: Forest plots of Xpert MTB/RIF diagnostic accuracy. The figure shows forest plots of Xpert MTB/RIF sensitivity and specificity in reference to 3 culture-based reference standards for individual studies. All reference standards classify patients as having TB if $\geq 1$ positive culture was confirmed as $M$. tuberculosis by speciation testing. To be classified as not having TB, patients were required to have no positive and at least 1) two negative cultures on two different sputum specimens (Standard 1); 2) two negative cultures on the same or different sputum specimens (Standard 2); or 3) at least one negative culture (Standard 3). Figure S6. TB-LAMP vs. Xpert MTB/RIF: Forest plots of TB-LAMP diagnostic accuracy. The figure shows forest plots of TB-LAMP sensitivity and specificity in reference to 3 culture-based reference standards for individual studies. All reference standards classify patients as having TB if $\geq 1$ positive culture was confirmed as M. tuberculosis by speciation testing. To be 
classified as not having TB, patients were required to have no positive and at least 1) two negative cultures on two different sputum specimens (Standard 1); 2) two negative cultures on the same or different sputum specimens (Standard 2); or 3) at least one negative culture (Standard 3). Figure S7. TBLAMP vs. Xpert MTB/Rif: Forest plots of sensitivity difference. The figure shows forest plots of the sensitivity difference between TB-LAMP and Xpert MTB/Rife for individual studies. The sensitivity of both tests was calculated in reference to 3 culture-based reference standards. All reference standards classify patients as having TB if $\geq 1$ positive culture was confirmed as $M$. tuberculosis by speciation testing. To be classified as not having TB, patients were required to have no positive and at least 1) two negative cultures on two different sputum specimens (Standard 1); 2) two negative cultures on the same or different sputum specimens; or 3) at least one negative culture (Standard 3). Visual inspection of forest plots and statistical testing indicate minimal heterogeneity with Standard 1 $\left(P^{2} 0 \%, p=0.41\right)$, some heterogeneity with Standard $2\left(P^{2} 34 \%, p=\right.$ $0.18)$, and significant heterogeneity with Standard $3\left(I^{2} 55 \%, p=0.03\right)$. Figure S8. TB-LAMP vs. Xpert MTB/Rif ${ }^{\oplus}$ : Forest plots of specificity difference. The figure shows forest plots of the specificity difference between TB-LAMP and Xpert MTB/Rif ${ }^{\oplus}$ for individual studies. The specificity of both tests was calculated in reference to 3 culturebased reference standards. All reference standards classify patients as having TB if $\geq 1$ positive culture was confirmed as $M$. tuberculosis by speciation testing. To be classified as not having TB, patients were required to have no positive and at least 1) two negative cultures on two different sputum specimens (Standard 1); 2) two negative cultures on the same or different sputum specimens (Standard 2); or 3) at least one negative culture (Standard 3). Visual inspection of forest plots and statistical testing indicate minimal heterogeneity with Standard 1 $\left(I^{2} 28 \%, p=0.25\right)$ and Standard $2\left(l^{2} 37 \%, p=0.16\right)$, but significant heterogeneity with Standard $3\left(R^{2} 72 \%, p=0.001\right)$. Figure $\mathbf{S 9}$.

Proportion of indeterminate TB-LAMP results. The figure shows a forest plot of the proportion of indeterminate TB-LAMP results among all adults for individual studies. Visual inspection of forest plots and statistical testing indicate minimal heterogeneity ( $P^{2} 28 \%, p$ $=0.25$. . Table S1. Patients included for analysis. Table S2. Signaling questions for QUADAS-2 domains. Table S3. TB-LAMP as an alternative test for smear microscopy: Exploration of heterogeneity. (DOCX $770 \mathrm{~kb}$ )

\section{Abbreviations}

AFB: Acid fast bacilli; Cl: Confidence interval; DEMO: Demonstration study; DNA: Deoxyribonucleic acid; EVAL: Evaluation study; FIND: Foundation for Innovative Diagnostics; FM: Fluorescence microscopy; HIV: Human immunodeficiency virus; HSROC: Hierarchical summary reciever operating characteristic; LAMP: Loop-mediated isothermal amplification; MTB: Mycobacterium tuberculosis; NTM: Non-tuberculous mycobacteria; QUADAS: Quality Assessment of Studies of Diagnostic Accuracy for Systematic Reviews; RFA: Request for Applications; RIF: Rifampin; TB: Tuberculosis; UV: Ultraviolet; WHO: World Health Organization; ZN: ZiehlNeelsen

\section{Acknowledgements \\ Not applicable.}

\section{Funding}

Funding for this study was provided by the Global TB Programme of the World Health Organization. The funder of the study (WHO) established the review questions to be addressed and reviewed the study protocol. The funder had no role in data collection, data analysis, data interpretation, or writing of the manuscript. The funder did incorporate a report of these results into their published guidelines on the use of TB-LAMP.

\section{Availability of data and materials}

The data that support the findings of this study are available from Eiken, but restrictions apply to the availability of these data, which were used at the discretion of Eiken for the current study, and so are not publicly available. Data are however available from the authors upon reasonable request and with permission of Eiken.

\section{Authors' contributions}

$\mathrm{PS}, \mathrm{KF}$, and LS conducted the literature search. All authors contributed to the design of the study. PS and KF developed the figures for the manuscript. Any additional data collection from Eiken or other sources was conducted by PS, KF, LS, and CG. PS KF LS CG collaborated on data analysis. Data were interpreted by PS, KF, LS, and AC. PS and KF wrote the initial version of the manuscript and all authors contributed to review and editing of the final version. The corresponding author had full access to all the data and had final responsibility for the decision to submit for publication. All authors read and approved the final manuscript.

\section{Ethics approval and consent to participate}

Ethics approval for this study was waived (University of California, San Francisco Internal Review Board) as it involved analysis only of previously collected de-identified data received by the authors from Eiken and from individual study sites.

\section{Consent for publication}

Not applicable.

\section{Competing interests}

The authors declare they have no competing interests.

\section{Publisher's Note}

Springer Nature remains neutral with regard to jurisdictional claims in published maps and institutional affiliations.

\section{Author details}

${ }^{1}$ Division of Pulmonary and Critical Care Medicine, University of California San Francisco and Zuckerberg San Francisco General Hospital, 1001 Potrero Avenue, 5K1, San Francisco, CA 94110, USA. ${ }^{2}$ Curry International Tuberculosis Center, University of California San Francisco, San Francisco, CA, USA. ${ }^{3}$ Division of Infectious Diseases, Oregon Health \& Science University, 3188 SW Sam Jackson Park Road, Mail Code: L457, Portland, OR 97239, USA. ${ }^{4}$ Faculty of Epidemiology and Population Health, London School of Hygiene and Tropical Medicine, Room G30, Keppel Street, London WC1E 7HT, UK.

Received: 21 September 2017 Accepted: 5 March 2019

Published online: 19 March 2019

\section{References}

1. World Health Organization: Automated real-time nucleic acid amplicfication technology for rapid and simultaneous detection of tuberculosis and rifampicin resistance: Xpert MTB/RIF assay for the diagnosis of pulmonary and extrapulmonary TB in adults and children. In.; 2013.

2. Notomi T, Okayama H, Masubuchi H, Yonekawa T, Watanabe $\mathrm{K}$, Amino N, Hase T. Loop-mediated isothermal amplification of DNA. Nucleic Acids Res. 2000;28(12):E63.

3. Iwamoto T, Sonobe T, Hayashi K. Loop-mediated isothermal amplification for direct detection of mycobacterium tuberculosis complex, M. Avium, and M. Intracellulare in sputum samples. J Clin Microbiol. 2003;41(6):2616-22.

4. Sethi S, Singh S, Dhatwalia SK, Yadav R, Mewara A, Singh M, Tewari R, Sharma M. Evaluation of in-house loop-mediated isothermal amplification (LAMP) assay for rapid diagnosis of M. Tuberculosis in pulmonary specimens. J Clin Lab Anal. 2013;27(4):272-6.

5. Kaewphinit T, Arunrut N, Kiatpathomchai W, Santiwatanakul S, Jaratsing P, Chansiri K. Detection of mycobacterium tuberculosis by using loopmediated isothermal amplification combined with a lateral flow dipstick in clinical samples. Biomed Res Int. 2013;2013:926230.

6. Aryan E, Makvandi M, Farajzadeh A, Huygen K, Bifani P, Mousavi SL, Fateh A, Jelodar A, Gouya MM, Romano M. A novel and more sensitive loopmediated isothermal amplification assay targeting IS6110 for detection of mycobacterium tuberculosis complex. Microbiol Res. 2010;165(3):211-20.

7. Mitarai S, Okumura M, Toyota E, Yoshiyama T, Aono A, Sejimo A, Azuma Y, Sugahara K, Nagasawa T, Nagayama N, et al. Evaluation of a simple loopmediated isothermal amplification test kit for the diagnosis of tuberculosis. Int J Tuberc Lung Dis. 2011;15(9):1211-7 i.

8. Higgins J, Green S, Cochrane Collaboration., Wiley InterScience (Online service). Cochrane handbook for systematic reviews of interventions. In: Cochrane book series. Chichester, England; Hoboken, NJ: Wiley-Blackwell; 2008. 1 online resource (xxi, 649 p.). 
9. Whiting PF, Rutjes AW, Westwood ME, Mallett S, Deeks JJ, Reitsma JB, Leeflang MM, Sterne JA, Bossuyt PM, Group Q. QUADAS-2: a revised tool for the quality assessment of diagnostic accuracy studies. Ann Intern Med. 2011;155(8):529-36.

10. Higgins JP, Thompson SG. Quantifying heterogeneity in a meta-analysis. Stat Med. 2002;21(11):1539-58.

11. Higgins JP, Thompson SG, Deeks JJ, Altman DG. Measuring inconsistency in meta-analyses. BMJ. 2003;327(7414):557-60.

12. Gray CM, Katamba A, Narang P, Giraldo J, Zamudio C, Joloba M, Narang R, Paramasivan CN, Hillemann D, Nabeta P, et al. Feasibility and operational performance of tuberculosis detection by loop-mediated isothermal amplification platform in decentralized settings: results from a multicenter study. J Clin Microbiol. 2016;54(8):1984-91.

13. Nliwasa M, MacPherson P, Chisala P, Kamdolozi M, Khundi M, Kaswaswa K, Mwapasa M, Msefula C, Sohn H, Flach C, et al. The sensitivity and specificity of loop-mediated isothermal amplification (LAMP) assay for tuberculosis diagnosis in adults with chronic cough in Malawi. PLoS One. 2016;11(5):e0155101.

14. Kaku T, Minamoto F, D'Meza R, Morose W, Boncy J, Bijou J, Geffrard H, Yoshida M, Mori T. Accuracy of LAMP-TB method for diagnosing tuberculosis in Haiti. Jpn J Infect Dis. 2016;69(6):488-92.

15. World Health Organization. High-priority target product profiles for new tuberculosis diagnostics: report of a consensus meeting. Geneva: World Health Organization; 2014.

16. Steingart KR, Schiller I, Horne DJ, Pai M, Boehme CC, Dendukuri N. Xpert(R) MTB/RIF assay for pulmonary tuberculosis and rifampicin resistance in adults. Cochrane Database Syst Rev. 2014. https://doi.org/10.1002/14651858. CD009593.pub3.

17. World Health Organization. The use of loop-mediated isothermal amplification (TB-LAMP) for the diagnosis of pulmonary tuberculosis. Policy guidance. Geneva: World Health Organization; 2016.

18. Sohn H. Cost, affordability, and cost-effectiveness of TB-LAMP assay. In: Report to WHO Guideline Development Group Meeting on TB-LAMP Assay. Edn. Geneva: World Health Organization; 2016.

Ready to submit your research? Choose BMC and benefit from:

- fast, convenient online submission

- thorough peer review by experienced researchers in your field

- rapid publication on acceptance

- support for research data, including large and complex data types

- gold Open Access which fosters wider collaboration and increased citations

- maximum visibility for your research: over $100 \mathrm{M}$ website views per year

At $\mathrm{BMC}$, research is always in progress.

Learn more biomedcentral.com/submissions 